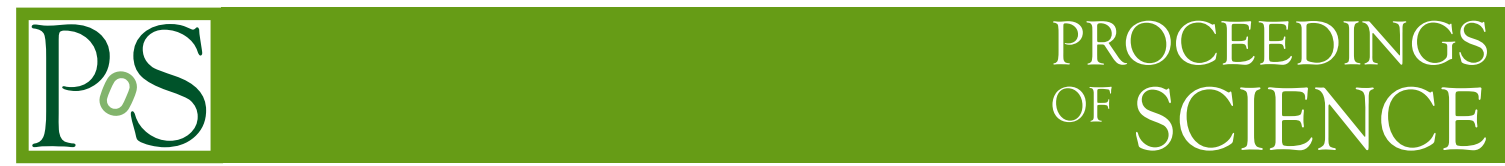

\title{
Top mass measurement at LHC
}

\author{
Anne-Isabelle Etienvre \\ CEA-Saclay, Dapnia/Service de Physique des Particules, F-91191 Gif-sur-Yvette Cedex, France \\ E-mail: anne-isabelle.etienvre@cea.fr
}

The Large Hadron Collider (LHC) will be a "top factory", since about 8 millions of ttbar events will be produced in 100 days of running at low luminosity $\left(10^{33} \mathrm{~cm}^{-2} \mathrm{~s}^{-1}\right)$, which corresponds to an integrated luminosity equal to $10 \mathrm{fb}^{-1}$. This large amount of data will allow an accurate measurement of the top quark mass, with a precision at the level of $1 \mathrm{GeV} / \mathrm{c}^{2}$, dominated by systematic uncertainties. The physics goals of this accuracy will be recalled. The principle of this measurement in all decay channels of the ttbar production will be described. The estimate of the main systematic uncertainties (energy scale, final state radiation) in these several channels, assuming nominal running conditions, will be given.

International Workshop on Top Quark Physics

January 12-15, 2006

Coimbra, Portugal 


\section{Introduction}

At LHC, the top quark will be produced mainly in pairs through the hard process $g g \rightarrow t \bar{t}$ (90\%) and $q \bar{q} \rightarrow t \bar{t}$ (10\%); the corresponding cross-section, at the next-to-leading order, is equal to $833 \mathrm{pb}$ : therefore, we expect roughly 8 million $t \bar{t}$ pairs to be produced with 100 days at low luminosity (corresponding to an integrated luminosity of $10 \mathrm{fb}^{-1}$ ).

Within the SM, the top quark decays almost exclusively (99.9\%) into a W boson and a b-quark. Depending on the decay mode of the $\mathrm{W}$ bosons, the $t \bar{t}$ events can be classified into three channels : the lepton plus jets channel (B.R. $\simeq 30 \%$, considering only electrons and muons), the dilepton channel (B.R. $\simeq 5 \%$ ) and the fully hadronic channel (B.R. $\simeq 44 \%$ ). The top mass measurement can be performed in these three channels : the different methods are explained, with their advantages, their disadvantages, and their corresponding systematic errors.

\section{Motivations for an accurate measurement of the top mass}

\subsection{Theoretical motivations}

Electroweak precision observables in the Standard Model (SM) and the Minimal Supersymetric Standard Model (MSSM) depend on the value of the top mass ; therefore, a high accuracy in the top mass measurement is needed for concistency tests of the Standard Model, constraints on the Higgs mass within the SM and a high sensitivity to physics beyond the Standard Model. The most important top mass dependence contribution to the Electroweak observables arises via the one-loop radiative correction term $\Delta \mathrm{r}$ [1], related to the $\mathrm{W}$ mass through the following relation : $m_{W}^{2}=\frac{\pi \alpha}{\sqrt{2} G_{F} \sin ^{2} \Theta_{W}}(1+\Delta r)$. The top mass arises in $\Delta r$ via terms proportional to $m_{t}^{2} / m_{Z}^{2}$, while the Higgs mass gives rise to terms proportional to $\log m_{H} / m_{Z}$ : therefore, the dependence on the Higgs mass is much weaker than the dependence on the top mass.

The relation thus obtained is used as an indirect estimate of the Higgs boson mass, relying on W boson and top quark masses measurements as accurate as possible : $m_{H}=91_{-32}^{+45} \mathrm{GeV} / \mathrm{c}^{2}, m_{H}<$ $186 \mathrm{GeV} / \mathrm{c}^{2}$ at $95 \%$ C.L., for the current value of the top mass $\left(m_{\text {top }}=172.7 \pm 2.9 \mathrm{GeV} / \mathrm{c}^{2}\right)$ [2]. The allowed region in the $\left(m_{W}, m_{t}\right)$ plane is displayed in Figure 1, for different Higgs boson masses, in the SM and in the MSSM.

\subsection{What would bring a precision on the top mass of the order of $1 \mathrm{GeV} / \mathrm{c}^{2}$ ?}

In order to ensure a similar contribution to the indirect measurement of the Higgs mass, the precision on $m_{W}$ and $m_{t}$ must fulfil the following relation : $\Delta m_{t} \simeq 0.710^{-2} \Delta m_{W}$. At LHC, we expect to reach an accuracy of $15 \mathrm{MeV} / c^{2}$ on $m_{W}$ and $1 \mathrm{GeV} / c^{2}$ on $m_{t}$. With these precision measurements, the relative precision on a Higgs boson mass of $115 \mathrm{GeV} / \mathrm{c}^{2}$ would be of the order of $18 \%$ [4].

\section{Definition of the systematic errors on the top mass measurement}

For the top mass analyses presented here, performed within ATLAS or CMS, several systematic uncertainties have been estimated. The main sources of errors, common to several analyses, are briefly described below. 


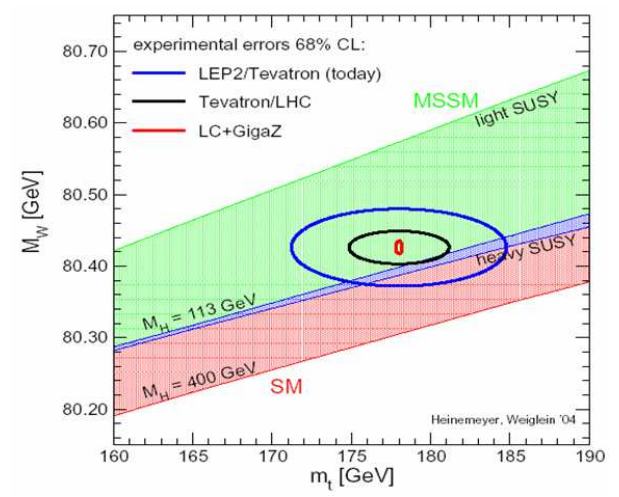

Figure 1: Current experimental results for $m_{W}$ and $m_{t o p}$, and expected accuracies at the next generation of colliders, with lines of constant Higgs boson masses in the Standard Model, and in the MSSM [3]

\subsection{Jet energy scale}

When the top quark is reconstructed via its hadronic decay $(\mathrm{t} \rightarrow \mathrm{Wb} \rightarrow \mathrm{jjb})$, the accuracy of the measurement of its mass relies on a precise knowledge of the energy calibration for both light jets and b-jets. The energy of the two light jets can be calibrated precisely event by event using an in-situ calibration based on the W mass constraint [5], while the b-jet energy scale has to be calibrated independently : therefore, their contributions to systematic errors are always estimated separately.

A jet energy scale calibration at the level of $1 \%$, for both light jets and b-jets, should be reached at LHC : the corresponding errors on the top mass measurement given below correspond to this level of precision. The estimation of an absolute jet energy scale uncertainty has been carried out applying different miscalibration coefficients to the reconstructed jet energies ; a linear dependence has been observed.

\subsection{Initial and final state radiation}

The presence of initial state radiation (ISR) of incoming partons and final state radiation (FSR) from the top decay products has an impact on the top mass measurement. In order to estimate the uncertainty due to these radiations, the top mass has been determined with ISR (FSR) switched on, at the generator level, and ISR (FSR) switched off. The systematic uncertainty on the top mass is taken to be $20 \%$ of the corresponding mass shifts : this should be a conservative estimate, assuming that ISR and FSR are known at a level of order of $10 \%$ [8].

\section{3 b-quark fragmentation}

The systematic error due to an imperfect knowledge of the b-quark fragmentation has been estimated by varying the Peterson parameter of the fragmentation function (equal to -0.006) within its experimental uncertainty $(0.0025)$ : the consecutive shift on the top mass is taken as the systematic error on the top mass. 


\begin{tabular}{||c|c|c||}
\hline Process & Cross section $(\mathrm{pb})$ & Number of events @ $10 \mathrm{fb}^{-1}$ (millions) \\
\hline \hline Signal & 250 & 2.5 millions \\
\hline \hline$b \bar{b} \rightarrow l v+$ jets & $2.210^{6}$ & $2210^{3}$ \\
\hline$W+$ jets $\rightarrow l v+$ jets & $7.810^{3}$ & 78 \\
\hline$Z+$ jets $\rightarrow l^{+} l^{-}+$jets & $1.210^{3}$ & 12 \\
\hline$W W \rightarrow l v+$ jets & 17.1 & 0.17 \\
\hline$W Z \rightarrow l v+$ jets & 3.4 & 0.034 \\
\hline$Z Z \rightarrow l^{+} l^{-}+$jets & 9.2 & 0.092 \\
\hline \hline
\end{tabular}

Table 1: Main backgrounds to the lepton $(l=e, \mu)+$ jets $t \bar{t}$ signal

\subsection{Background}

The background of the top quark reconstruction is dominated by wrong combinations in $t \bar{t}$ events themselves (FSR, wrong association of the $\mathrm{W}$ to the corresponding b-jet,..). Varying the background shape and size in the fitting procedure of the top mass distribution gives access to the resulting uncertainty on the top mass measurement.

\section{Top mass measurement in the lepton + jets channel}

The lepton plus jets channel will provide a large and clean sample of $t \bar{t}$ events and is probably the most promising channel for an accurate measurement of the top mass. The main backgrounds are summarized in Table 1, with their corresponding cross sections and expected number of events at $10 \mathrm{fb}^{-1}$. Before any selection, the signal over background ratio is of the order of $10^{-4}$. Events are selected by requiring one isolated lepton (electron or muon) with $p_{T}>20 \mathrm{GeV} / \mathrm{c}$ and $|\eta|<2.5$, $E_{T}^{\text {miss }}>20 \mathrm{GeV} / c^{2}$, and at least 4 jets with $p_{T}>40 \mathrm{GeV} / \mathrm{c}$ and $|\eta|<2.5$, of which two of them are required to be tagged as b-jets. Jets used for these analysis are reconstructed with a $\Delta R=0.4^{1}$ cone algorithm. After these cuts, $\mathrm{S} / \mathrm{B}$ becomes much more favourable $: \mathrm{S} / \mathrm{B} \simeq 30$.

\subsection{Top mass measurement using the hadronic top decay ([6], [7], [9])}

The top mass is estimated here from the reconstruction of the invariant mass of a three-jet system : the two light jets from the $\mathrm{W}$ and one of the two b-jets. The determination of this combination of three jets proceeds in two steps : the choice of the two light jets, and the choice of the $\mathrm{b}$-jet associated to the reconstructed hadronic W.

\subsubsection{Reconstruction of the hadronic W [6]}

Events kept after the selection described above have at least two light jets above a given threshold on their transverse momentum. In a first step, we select the hadronic W candidates in a mass window of $\pm 5 \sigma_{m j j}$ around the peak value of the distribution of the invariant mass of the light jet pairs, made with events with only two light jets $\left(\sigma_{m j j}\right.$ is the width of this distribution).

In order to reduce the incidence of a light-jet energy mis-measurement (due to the energy lost out

$$
{ }^{1} \Delta R=\sqrt{\Delta \Phi^{2}+\Delta \eta^{2}}
$$


of cone) on the precision of the top mass measurement, an in-situ calibration of these jets is performed, through a $\chi^{2}$ minimization procedure ([5], [7]). This minimization is applied event by event, for each light-jet pair combination. The expression of $\chi^{2}$, given by equation (4.1), is the sum of three terms : the first (and leading) one corresponds to the constrain of the jet pair invariant mass $m_{j j}$ to the PDG W mass $\left(m_{W}\right)$; the others correspond to the jet energy correction factors, $\alpha_{i}(i=1,2)$, to be determined by this minimization $\left(\sigma_{i}(i=1,2)\right.$ is the resolution on the light jet energy).

$$
\chi^{2}=\frac{\left(m_{j j}\left(\alpha_{1}, \alpha_{2}\right)-m_{W}\right)^{2}}{\Gamma_{W}^{2}}+\frac{\left(E_{j 1}\left(1-\alpha_{1}\right)\right)^{2}}{\sigma_{1}^{2}}+\frac{\left(E_{j 2}\left(1-\alpha_{2}\right)\right)^{2}}{\sigma_{2}^{2}}
$$

The $\chi^{2}$ is minimized, event by event, for each light jet pair ; the light jet pair $j_{1}, j_{2}$ corresponding to the minimal $\chi^{2}$ is kept as the hadronic $\mathrm{W}$ candidate. This minimization procedure also leads to the corresponding energy correction factors $\alpha_{1}, \alpha_{2}$. The hadronic $\mathrm{W}$ is then reconstructed with the light jets chosen by this $\chi^{2}$ minimization.

\subsubsection{Top mass reconstruction}

Several methods have been investigated to choose the b-jet among the two candidates, and the one giving the highest purity has been kept : the b-jet associated to the hadronic $\mathrm{W}$ is the one leading to the highest $p_{T}$ for the top.

The reconstructed three jets invariant mass is shown in Figure 2, : the mass peak (176.1 \pm 0.6 $\left.\mathrm{GeV} / c^{2}\right)$ is in reasonable agreement with the generated value $\left(175 \mathrm{GeV} / c^{2}\right)$; the width is equal to $11.9 \pm 0.7 \mathrm{GeV} / c^{2}$. The overall efficiencies and purities, with respect to lepton + jets events, are summarized in Table 2 : we expect with this method 64000 events at $10 \mathrm{fb}^{-1}$, corresponding to a statistical error equal to $0.05 \mathrm{GeV} / c^{2}$.

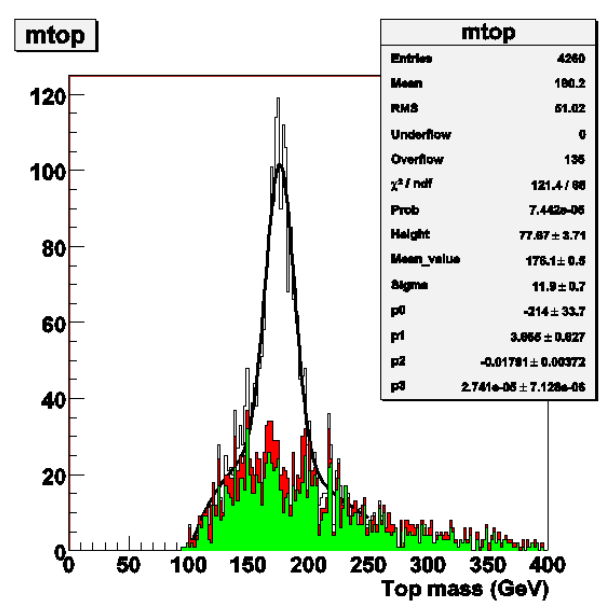

Figure 2: Top mass distribution, with the contribution from wrong W combinations, in green, and, in red, from wrong b-jet associations. This analysis has been performed using the MC@NLO generator and the full simulation of the ATLAS detector. 


\begin{tabular}{||c|c|c|c|c|}
\hline & Efficiency (\%) & $\mathrm{b}$ purity (\%) & $\mathrm{W}$ purity (\%) & Top purity (\%) \\
\hline full mass window & $2.70 \pm 0.005$ & $56.0 \pm 0.9$ & $63.2 \pm 0.9$ & $40.5 \pm 0.9$ \\
mass window within $\pm 3 \sigma_{m_{\text {top }}}$ & $1.82 \pm 0.04$ & $69.1 \pm 0.8$ & $75.8 \pm 0.8$ & $58.6 \pm 0.8$ \\
\hline
\end{tabular}

Table 2: Total efficiency and $W, b$ and top purity of the final selected events (MC@NLO, full simulation of the ATLAS detector), with respect to lepton (electron, muon) + jets events

\subsubsection{W + jets background contribution}

The dominant remaining background to lepton + jets $t \bar{t}$ events comes from $\mathrm{W}+$ jets events. The contribution to the top mass measurement is negligible : the values of the mass peak $(176.1 \pm$ $0.6 \mathrm{GeV} / c^{2}$ for signal only, $176.2 \pm 0.6 \mathrm{GeV} / c^{2}$ for signal plus background) and of the width (11.9 $\pm 0.7 \mathrm{GeV} / c^{2}$ for signal, $12.1 \pm 0.7 \mathrm{GeV} / c^{2}$ for signal plus background ) are identical.

\subsection{Top mass measurement using a kinematic fit [7]}

An alternative method for the top mass measurement in the lepton plus jets channel consists in reconstructing the entire $t \bar{t}$ final state, in order to reduce the systematic error due to FSR. The hadronic part is reconstructed in a similar way to the previous section. The leptonic side can not be directly reconstructed due to the presence of the undetected neutrino, but can be estimated in three steps :

- $p_{T}(v)=E_{T}^{m i s s}$

- $p_{z}(v)$ is obtained by constraining the invariant mass of the lepton-neutrino system to the PDG W mass value : this kinematic equation leads to two $p_{z}(v)$ solutions

- the remaining b-jet is associated to the reconstructed $\mathrm{W}$

The top mass determination is performed through a kinematic fit, relying on a $\chi^{2}$ based on mass constraints $\left(m_{j j}=m_{W}^{P D G}=m_{l v} ; m_{j j b}=m_{l v b}\right)$ and kinematic constraints (energy and direction of leptons and jets can vary within their resolutions). The minimization of this $\chi^{2}$ is performed event by event, for the two $p_{z}(v)$ solutions : the one giving the lower $\chi^{2}$ is kept. The top mass is determined as the linear extrapolation of $m_{\text {top }}\left(\chi^{2}\right)$ for $\chi^{2}=0$.

With an efficiency equal to $1.1 \%$, we expect with this method 26000 events at $10 \mathrm{fb}^{-1}$, corresponding to a statistical error equal to $0.1 \mathrm{GeV} / c^{2}$. This analysis has been performed using a fast simulation of the ATLAS detector, and will be checked with a full simulation. Another approach, developped by CMS, is described in J.Heyninck's contribution to the TOP2006 Workshop.

\subsection{Top mass measurement using large $p_{T}$ top events ([7] [10])}

Thanks to the large amount of $t \bar{t}$ events produced at LHC, a subsample of lepton + jets $t \bar{t}$ events, where the top quarks have a $p_{T}$ greater than $200 \mathrm{GeV} / \mathrm{c}$, can be studied. The interest of such events is that the top and the anti-top are produced back-to-back in the laboratory frame, so that their daughters will appear in distinct hemispheres of the detector : therefore, the combinatorial background should be strongly reduced. 
Because of the high $p_{T}$ (top), the three jets in one hemisphere tend to overlap. To overcome this problem, the top quark is reconstructed in a large calorimeter cone ( $\Delta R$ in $[0.8-1.8])$, around the top quark direction.

A strong dependence of the reconstructed top mass with the cone size has been observed and can be attributed to the Underlying Events (UE) contribution, evaluated to $45 \mathrm{MeV}$ in a $0.1 \mathrm{X} 0.1$ calorimeter tower with the full simulation of the ATLAS detector. After UE subtraction, the top mass is independent of the cone size, but lower than the generated top mass by $25 \%$, as can be seen in Figure 3. A mass scale recalibration, based on the hadronic W, is then applied and leads to an average top mass value consistent with the generated value (see Figure 3 ).

With an efficiency equal to $2 \%$ with respect to this subsample, we expect with this method 3600 events at $10 \mathrm{fb}^{-1}$, corresponding to a statistical error equal to $0.2 \mathrm{GeV} / \mathrm{c}^{2}$.
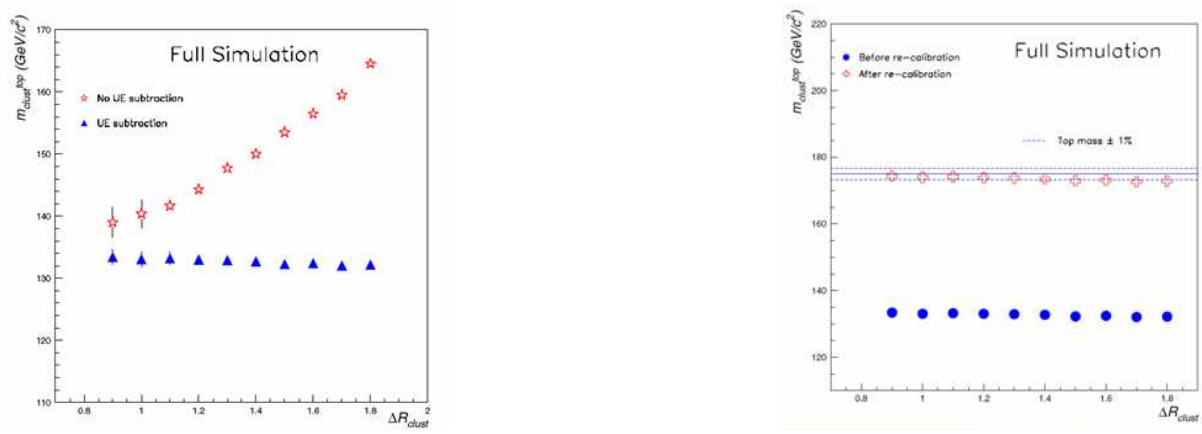

Figure 3: Fitted top mass reconstructed in a large calorimeter cluster as a function of the cluster size, for a subsample of events with $p_{T}$ (top) $>200 \mathrm{GeV} / \mathrm{c}$, before and after UE subtraction, on the left. The plot on the right shows the effect of the mass scale recalibration. This analysis has been performed using the PYTHIA generator for signal, and the full simulation of the ATLAS detector.

\subsection{Systematic uncertainties on the top mass measurement in the lepton + jets channel}

The systematic uncertainties on the top mass measurement are summarized in Table 3, for the three methods explained above. It is possible to get rid of the error due to the light jet energy scale thanks to the in-situ calibration ; the dominant contribution comes from the FSR and the b-jet energy scale.

\section{Top mass measurement in leptonic final states with $\mathrm{J} / \Psi$ ([11])}

A last top mass determination can be carried out in the lepton+jets channel where a $\mathrm{J} / \Psi$ arises from the b-quark associated to the leptonic decaying W (Figure 4). The large mass of the J/ $\Psi$ induces a strong correlation with the top mass, as will be shown below. To solve the ambiguity between the two b-jets, a charge tagging of the $\mathrm{b}$ decaying to $\mathrm{J} / \Psi$ is applied, requiring a muon of the same electric charge as the isolated lepton in the other b-jet. The overall branching ratio $\left(5.310^{-5}\right)$ is low : therefore, this analysis will be performed at high luminosity $\left(10^{34} \mathrm{~cm}^{-2} \mathrm{~s}^{-1}\right)$. Four-lepton events are selected by requiring an isolated lepton with $p_{T}>15 \mathrm{GeV}$ and $|\eta|<2.4$, and three non-isolated muons with $p_{T}>4 \mathrm{GeV} / \mathrm{c}$ and $|\eta|<2.4$, with the invariant mass of two of them 


\begin{tabular}{||c|c|c|c||}
\hline Source of uncertainty & $\begin{array}{c}\text { Hadronic top } \\
\delta m_{\text {top }}\left(\mathrm{GeV} / \mathrm{c}^{2}\right)\end{array}$ & $\begin{array}{c}\text { Kinematic fit } \\
\delta m_{t o p}\left(\mathrm{GeV} / \mathrm{c}^{2}\right)\end{array}$ & $\begin{array}{c}\text { High } p_{T} \text { top sample } \\
\delta m_{\text {top }}\left(\mathrm{GeV} / \mathrm{c}^{2}\right)\end{array}$ \\
\hline Light jet energy scale (1\%) & 0.2 & 0.2 & \\
b-jet energy scale (1\%) & 0.7 & 0.7 & 0.3 \\
b-quark fragmentation & 0.1 & 0.1 & 0.1 \\
ISR & 0.1 & 0.1 & 0.1 \\
FSR & 1. & 0.5 & 0.9 \\
Combinatorial background & 0.1 & 0.1 & 1.3 \\
Mass rescaling & & & 1.6 \\
UE estimate $( \pm 10 \%)$ & & 0.9 & 0.2 \\
\hline Total & 1.3 & 0.1 & \\
\hline Statistical error & 0.05 & &
\end{tabular}

Table 3: Systematic errors on the top mass measurements, in the lepton + jets channel, for the three methods described above

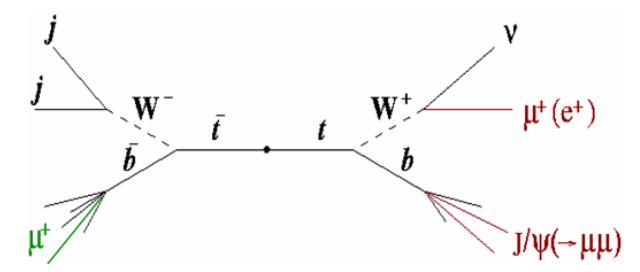

Figure 4: Diagram of the $t \bar{t}$ decay to semi-leptonic final state with $\mathrm{J} / \Psi$.

being consistent with the $\mathrm{J} / \Psi$ mass. These cuts reduce the external background to a negligible contribution, and lead to an efficiency of $30 \%$, corresponding to 1000 events per year at high luminosity.

An example of the $1 \mathrm{~J} / \Psi$ mass distribution is shown in Figure 5 : the background is essentially combinatorial, due to a wrong assignment of the $\mathrm{J} / \Psi$ to the corresponding isolated lepton. The measurement of the $1 \mathrm{~J} / \Psi$ mass, given by the peak position of the fitted distribution, has to be related to the generated top mass : these two masses are linearly correlated, as can be seen in Figure 5. A statistical error of the order of $0.5 \mathrm{GeV} / \mathrm{c}^{2}$ is expected on the $\mathrm{J} / \Psi$ mass measurement after 4 years at high luminosity, and the systematic error, dominated by the uncertainty on the b-quark fragmentation, is lower than $0.4 \mathrm{GeV} / \mathrm{c}^{2}$, which translates into a statistical error on the top mass measurement of $1 \mathrm{GeV} / \mathrm{c}^{2}$, and a systematic error lower than $0.8 \mathrm{GeV} / \mathrm{c}^{2}$.

\section{Top mass measurement in the dilepton channel [7]}

The dilepton channel is very clean, with a lower contribution of combinatorial background, but it can only provide an indirect top mass measurement, because of the presence of two undetected neutrinos in the final state. Events are selected requiring two leptons of opposite charge, with $p_{T}>$ $20 \mathrm{GeV} / \mathrm{c}$ and $|\eta|<2.5$, an $E_{T}^{\text {miss }}>40 \mathrm{GeV}$ and 2 b-jets with $p_{T}>25 \mathrm{GeV} / \mathrm{c}$ and $|\eta|<2.5$. After 

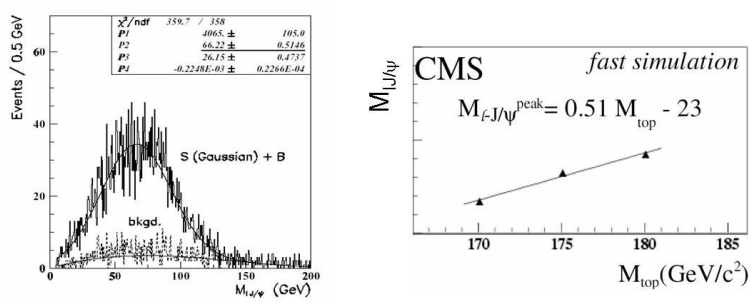

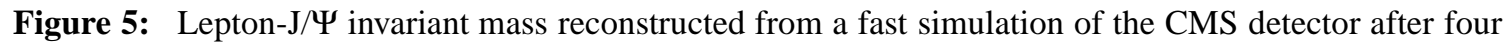
years at high luminosity (left). Correlation between the reconstructed $1 \mathrm{~J} / \Psi$ mass and the generated top quark mass (right). This study has been performed with a fast simulation of the CMS detector [9].

this selection, the ratio of signal over background is around 10 .

The final state reconstruction relies on a set of six equations for the six unknown components of momenta of neutrino and antineutrino, based on kinematic conservation laws and assuming a given top mass value. This set of equations can provide more than one solution; then, weights are computed from kinematic Monte Carlo distributions of three variables $\left(\cos \theta_{t o p}^{*}, E_{v}\right.$ and $\left.E_{\bar{v}}\right)$, and the solution corresponding to the highest weight is kept. This weight is computed for several input top masses, and the top mass estimator corresponds to the maximum mean weight.

With an efficiency of $6.5 \%, 20000$ events are expected at $10 \mathrm{fb}^{-1}$. The statistical error on the top mass measurement is negligible $\left(0.04 \mathrm{GeV} / \mathrm{c}^{2}\right)$. The systematic error, equal to $1.7 \mathrm{GeV} / \mathrm{c}^{2}$, is dominated by the uncertainty on the parton distribution function $\left(1.2 \mathrm{GeV} / \mathrm{c}^{2}\right)$.

\section{Top mass measurement in the all hadronic channel ([7])}

The main advantage of this channel is a full kinematic reconstruction of both sides, and its main disadvantage is the huge QCD multijet background : before any selection, the ratio of signal over background is very low $\left(10^{-8}\right)$. Events are selected requiring at least six jets with $p_{T}>40$ $\mathrm{GeV} / \mathrm{c}$, and $|\eta|<3$, and at least two b-jets with $p_{T}>40 \mathrm{GeV} / \mathrm{c}$, and $|\eta|<2.5$. The final state reconstruction proceeds in two steps : first, the choice of the two light jets pairs to form the two $\mathrm{W}$ bosons is performed through the minimization of a $\chi^{2}$ based on the $\mathrm{W}$ mass constraint. Both $\mathrm{W}$ candidates are then associated to the right b-jet minimizing a $\chi^{2}$ based on the equality of the top masses on both sides. In order to improve the signal over background ratio, the analysis can be restricted to a sample of high $p_{T}$ (>200 GeV/c) top and anti-top : this ratio is finally favourable ( $\mathrm{S} / \mathrm{B} \simeq 18) . \quad$ The top mass distribution is displayed in Figure 6 . The overall efficiency, within the $130-200 \mathrm{GeV} / \mathrm{c}^{2}$ top mass window, is equal to $0.08 \%$, corresponding to 3300 events at $10 \mathrm{fb}$ ${ }^{-1}$, and a statistical error equal to $0.18 \mathrm{GeV} / \mathrm{c}^{2}$. The systematic error, of the order of $3 \mathrm{GeV} / \mathrm{c}^{2}$, is dominated by the contribution of FSR $\left(2.8 \mathrm{GeV} / \mathrm{c}^{2}\right)$.

\section{Conclusion}

Various top mass measurement methods have been investigated, in all decay channels of the top quark. The very large sample of $t \bar{t}$ events that will be accumulated will allow a precision measurement after only one year of data taking at low luminosity $\left(10 \mathrm{fb}^{-1}\right)$ : the statistical error on 


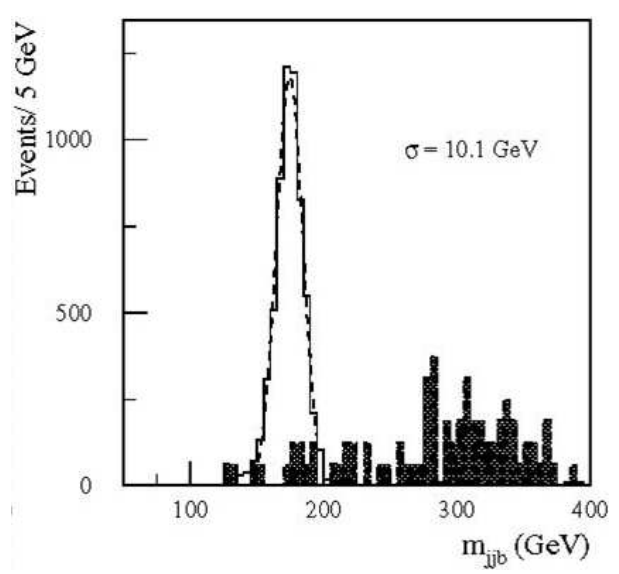

Figure 6: Top mass distribution in the all hadronic channel, for the high $p_{T}$ top sample. The shaded area corresponds to the remaining QCD background. This study has been performed with a fast simulation of the ATLAS detector [7].

the top mass is negligible in all these methods except the method involving leptonic final states with $J / \Psi$. These analyses are differently sensitive to the various sources of systematic uncertainties : therefore, this will allow reliable cross-checks between the various methods. The top quark mass should be measured at LHC with a precision of the order of $1 \mathrm{GeV} / \mathrm{c}^{2}$, in the lepton plus jets channel.

\section{References}

[1] A. Sirlin, $S U(2)_{L} X U(1)$ theory : a simple renormalization framework, Phys. Rev. D22, 971 (1980)

[2] The Tevatron Electroweak Working Group, hep-ex/0507091

[3] S. Heinemeyer, W. Hollik and G. Weiglein, Electroweak precision observables in the MSSM, hep-ph/041221

[4] Snowmass Working Group on Precision Electroweak measurements, Present and future Electroweak precision measurements and the indirect determination of the mass of the Higgs boson, eConf.

C010630 : P1WG1 (2001) [hep-ph/0202001]

[5] P. Roy, PhD Thesis, Blaise Pascal University, PCCFT0202 (2002)

[6] A.-I. Etienvre, J.-P. Meyer and J. Schwindling, Top quark mass measurement in the lepton plus jets channel using full simulation, ATLAS Internal note, ATL-PHYS-INT-2005-002 (2005)

[7] I. Borjanovic et al. Investigation of top mass measurements with the ATLAS detector at LHC, Eur.Phys.J, C39S2.63-90 (2005) [hep-ex/0403021]

[8] CERN Yellow Report, Top quark physics, 2000-004 [hep-ph/0003033]

[9] L. Sonnenschein, Top mass measurement in the lepton + jets channel, CMS Internal note, CMS-2001-001 (2001)

[10] I. Efthymiopoulos ATLAS Internal note, ATL-COM-PHYS-1999-050 (1999)

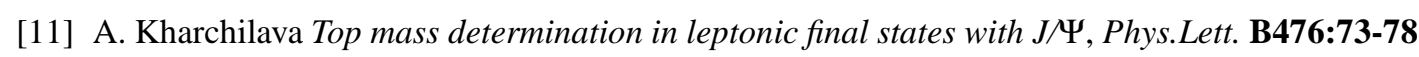
(2000) 